\title{
2 Land grabbing, Konflikte um Land und traditionelle Autoritäten - Stand der Forschung
}

Mit Blick auf die zentrale Frage, welcher Zusammenhang zwischen dem Handeln traditioneller Autoritäten und Konflikten um Zugang zu Land im Kontext von land grabbing besteht, bediene ich mich der Erkenntnisse aus drei Forschungsfeldern, zu (1) Konflikten im Zusammenhang mit land grabbing, (2) Land, sozialer Zugehörigkeit und Konflikten sowie (3) traditionellen Autoritäten als Intermediäre. In diesem Kapitel arbeite ich den aktuellen Stand der Forschung zu diesen Debatten auf, fasse die zentralen Erkenntnisse zusammen und zeige weiteren Forschungsbedarf auf.

Das Kapitel ist wie folgt strukturiert: Zunächst erarbeite ich einen Überblick über die aktuelle Forschung zu Konflikten im Kontext von land grabbing. Dabei beziehe ich mich vor allem auf Arbeiten aus der Politischen Ökologie als einem relevanten Forschungsfeld für die Analyse von Konflikten um den Zugang zu, die Kontrolle über und Rechte an Land. Insbesondere arbeite ich in diesem Abschnitt zentrale Erkenntnisse hinsichtlich der Gegenstände, Akteure und typischen Akteurskonstellationen in Konflikten im Kontext von land grabbing heraus und stelle aktuelle konzeptionelle Ansätze für die Analyse von Konflikten dar. Im zweiten Abschnitt fasse ich die wesentlichen Debatten zur Bedeutung sozialer Zugehörigkeit für den Zugang zu und die Kontrolle über Land in traditionellen Landverwaltungssystemen zusammen. Diesbezüglich zeige ich, inwiefern die koloniale Interpretation von »Tradition« die gegenwärtige Ausgestaltung traditioneller Landverwaltungssysteme bestimmt (hat). Ein zentrales Interesse gilt in diesem Abschnitt zudem der Wiederbelebung neotraditioneller Landrechtsansprüche in den letzten Jahrzehnten, dem Wechselverhältnis von Macht und Kontrolle über Land sowie dem Zusammenhang von sozialer Zugehörigkeit, Zugang zu Land und Konflikten. Im dritten Abschnitt erarbeite ich zentrale Erkenntnisse aus der Forschung zu traditionellen Autoritäten als Intermediäre. Ich gehe insbesondere 
auf zahlreiche Studien aus der historischen Forschung ein, die analysieren, inwiefern es sich bei der vermittelnden Rolle traditioneller Autoritäten um eine Zuschreibung aus der Kolonialzeit handelt. Darüber hinaus erläutere ich, in welchem Kontext traditionelle Autoritäten in jüngerer Zeit erneut als Intermediäre entdeckt wurden, welche Argumente diese Entwicklung begünstigten und welche Kritikpunkte diesbezüglich existieren.

\subsection{Land grabbing und Konflikte}

Land grabbing ist weder ein neues Phänomen (Alden Wily 2012), noch ein neuer Begriff (Barnes 1971). Dennoch hat das Thema in den letzten Jahren eine neue Prominenz erlangt. Spätestens die Veröffentlichung des GRAIN-Reports "Seized! The 2008 land grab for food and financial security « (GRAIN 2008), machte den Begriff land grabbing erneut berühmt und brachte dem Thema eine breite mediale und wissenschaftliche Aufmerksamkeit ein. Seither forschen Wissenschaftler*innen unterschiedlicher Fachdisziplinen und Forschungsfelder dazu.

Zahlreiche Beiträge betonen, dass land grabbing oftmals mit Konflikten einhergeht (vgl. u.a. Borras/Franco 2012; Hall et al. 2015; White et al. 2012; Wolford et al. 2013). Beiträge, die diese Konflikte erforschen liegen insbesondere in Form von Einzelfallstudien vor (Crawford/Botchwey 2017; Dietz 2019; Engels 2021a; Ndi/Batterbury 2017; Prause/Le Billon 2020). Einen wichtigen Beitrag zur systematischen Analyse von Konflikte, die in Verbindung mit land grabbing entstanden, haben Arbeiten aus der Politischen Ökologie geleistet. Mit ihrem Fokus auf die Untersuchung von Konflikten um die Verteilung natürlicher Ressourcen (Martinez-Alier 2002) hat die Politische Ökologie auch den aktuellen Stand der Forschung zu land grabbing und Konflikten maßgeblich bereichert.

\section{Forschung zu Konflikten um land grabbing und der Beitrag der Politischen Ökologie}

Die Politische Ökologie ist ein interdisziplinäres Forschungsfeld, das sich an einem marxistisch inspirierten Verständnis des Natur-Gesellschaftsverhältnisses orientiert (Watts/Peet 2004). Theoretisch beziehen sich Arbeiten aus diesem Feld vor allem auf kritische neo-marxistische, feministische, post-strukturalistische und post-koloniale Ansätze (Dietz/Engels 2017). Arbeiten aus der 
Politischen Ökologie betrachten Gesellschaft und Natur nicht als voneinander getrennt, sondern als wechselseitig konstitutive Sphären (Edelman et al. 2013; White et al. 2012; Wolford et al. 2013). Sie betonen den inhärent politischen Charakter von Konflikten um Natur und rücken in diesem Zusammenhang zugrundeliegende Macht- und Herrschaftsstrukturen in den Fokus der Analyse (Robbins 2012; Watts/Peet 2004). Aus Sicht der Politischen Ökologie sind Konflikte um Land und Rohstoffe durch Macht- und Herrschaftsverhältnisse strukturiert. Macht- und Herrschaftsverhältnisse sind ihrerseits maßgeblich dadurch konstituiert sind, wer Kontrolle über und Zugang zu Land und Rohstoffen hat (Wissen 2015). Damit bilden diese Arbeiten einen Gegensatz $\mathrm{zu}$ frühen Medienberichten, aktivistischen und wissenschaftlichen Beiträgen zu land grabbing und Konflikten, die weitgehend linear argumentierten (Dietz/Engels 2020). Eine typische Argumentationslinie ist, die großflächige Umnutzung von Land führe zur Vertreibung vorheriger Landnutzer*innen oftmals als homogene lokale Gemeinschaft (local community) gefasst - die daraufhin mit Widerstand reagierten, wodurch ein manifester Konflikt entstünde (kritisch dazu Borras/Franco 2013; Hall et al. 2015). Arbeiten aus der Politischen Ökologie brechen mit dieser linearen Logik und beziehen sich auf kontextabhängige, multi-skalare und relationale Erklärungsansätze. In der Analyse von Konflikten, die im Zusammenhang mit land grabbing stehen, berücksichtigen Arbeiten aus der Politischen Ökologie sowohl globale Transformationsprozesse und die damit einhergehenden sozial-räumlichen, ökologischen und strukturellen Veränderungen als auch das Handeln der involvierten Akteure (Borras/Franco 2013; Dietz/Engels 2020; Hall et al. 2015). In Bezug auf Letztere verweisen aktuelle Beiträge vermehrt darauf, dass vermeintlich homogene Gruppen (beispielsweise Landnutzer*innen) differenziert zu betrachten und Kategorien wie Klasse, Geschlecht, Alter, race, ethnische und politische Zugehörigkeit zu berücksichtigen seien (Escobar 2006; Hall et al. 2015; Kirst/Prause 2019; Porro 2010). Beiträge aus dem Feld der Politischen Ökologie betonen darüber hinaus den inhärent politischen Charakter der Aneignung, Verteilung und des Verbrauchs natürlicher Ressourcen. Diese Prozesse seien eng mit gesellschaftlichen Eigentums- und Kontrollverhältnissen sowie zugrundeliegenden Machtstrukturen verknüpft (Bryant/Bailey 1997).

Aus der Perspektive der Politischen Ökologie sind Konflikte in ihrem jeweiligen historischen Kontext verwurzelt, finden in sozialen Beziehungen Ausdruck, stehen aber auch in Zusammenhang mit übergreifenden globalen Transformationsprozessen und Machtverhältnissen (Peluso/Watts 2001: 5). 
Konflikte werden aus dieser Perspektive nicht als zwingend negativ oder zerstörerisch gewertet, sondern als Möglichkeit, gesellschaftlichen Wandel zu begünstigen (Le Billon 2015: 599). Um Potenziale von Konflikten im Kontext von land grabbing für gesellschaftlichen Wandel erkennen zu können, sei ein tieferes Verständnis der sozialen, ökologischen und politischen Vermittlung dieser Konflikte nötig. In Bezug auf die politische Vermittlung werfen aktuelle Forschungen zunehmend die Frage nach der Rolle des Staates im Zusammenhang mit land grabbing auf (Boone 2015; Engels/Dietz 2017; Lavers 2012; Robbins 2008). Insbesondere fordern sie die Darstellung früherer Beiträge heraus, die Staaten entweder als »Zielländer« oder »Gastgeberländer« (host countries) von land grabbing einstufte. Als »Zielländer « gelten Staaten, die zu schwach sind, um ihre Grenzen und ihr Territorium vor ausländischen Investoren $\mathrm{zu}$ »schützen«. Als »Gastgeberländer« gelten solche, die mittels staatlicher Unternehmen oder durch die Bereitstellung von Infrastruktur und Informationen land grabbing ermöglichen oder unterstützen (Hall et al. 2015). Aktuelle Analysen hinterfragen diese Sichtweise und sprechen sich gegen eine Konzeptualisierung des Staats als homogenen Akteur aus, der mit einer Stimme spreche und einer einheitlichen Handlungslogik folge. Sie fassen den Staat nicht als kohärente Einheit, sondern analysieren stattdessen das Zusammenspiel unterschiedlicher, in Konkurrenz um politischen Einfluss zueinander stehender Institutionen, Akteuren, Interessen und Gruppierungen. Denn staatliche Akteure seien je nach Kontext und Zeitpunkt immer auch zivilgesellschaftliche Akteure. Daraus leite sich die Relevanz ab, die Rolle des Staates immer in Bezug zum jeweiligen Kontext zu sehen. Denn ebenso wie vielfältige Sichtweisen und Interessen innerhalb des Staates existierten, gebe es auch vielfältige Beziehungen zwischen Staat und Gesellschaft (Hall et al. 2015: 477; Schenk 2018).

\section{Gegenstände, Akteure und Akteurskonstellationen in Konflikten um land grabbing}

Die bisherige Forschung verdeutlicht, dass in Konflikten, die im Zusammenhang mit land grabbing auftreten zahlreiche unterschiedliche Themen im Mittelpunkt stehen. Arturo Escobar (2006) schlägt eine Analyse der Konflikte anhand von drei zentralen zusammenhängenden Bereichen vor: Ökonomie, Ökologie und Kultur. Die Relevanz dieser drei Bereiche erklärt er über einen »dreifachen Wandel« (triple transformation), den die Kommodifizierung natürlicher Ressourcen nach sich zöge (Escobar 2006: 7). Mit dem Termi- 
nus des »dreifachen Wandels« beschreibt Escobar den Wandel einer diversifizierten lokalen, teilweise subsistenzorientierten und auf Eigenproduktion ausgerichteten Wirtschaft hin zu einer monetarisierten, marktorientierten Wirtschaft; den Wandel komplexer Ökosysteme in "moderne Formen von Natur« wie Plantagen und Monokulturen; und den Wandel ortsbezogener, lokaler Kulturen hin zu solchen, die vermehrt dominanten Kulturen des globalen Nordens gleichen (müssen). Aus dem dreifachen Wandel ließen sich unterschiedliche Konfliktthemen ableiten, die letztendlich alle mit Fragen gesellschaftlicher Verteilung zusammenhingen: Verteilung von Einkommen und Gewinn, von natürlichen Ressourcen und von sozialer Macht. Letztere bestimme maßgeblich den Handlungsspielraum der Akteure und habe einen Einfluss darauf, wie Mitbestimmung ausgestaltet ist und inwiefern marginalisierte Gruppen oder Minderheiten durch herrschende Akteure anerkannt, berücksichtigt und integriert werden (Escobar 2006).

Andere Arbeiten fassen Konflikte im Zusammenhang mit land grabbing als Konflikte um den Zugang zu und die Kontrolle über Land (Peluso/Lund 2011). Zugang beschreibt in diesem Kontext nicht den physischen Zugriff auf Land, sondern die Fähigkeit einen Nutzen daraus ziehen zu können (Ribot/Peluso 2003). Diese Fähigkeit hängt maßgeblich mit bestehenden Landrechten zusammen, die Besitzverhältnisse gestalten und bestimmte Akteure ermächtigen, Kontrolle über den Zugang zu Land auszuüben. Je nach Kontext können dies traditionelle Autoritäten, der Staat oder Individuen sein (Boone 2013, 2015). De jure Besitzverhältnisse allein - seien sie aus mündlich übermittelten Gewohnheitsrechten oder schriftlich festgehaltenen formalisierten Rechten abgeleitet - spiegeln den vielschichtigen Zugang zu Ressourcen jedoch in der Regel nicht ausreichend wider. Im Fokus der Analyse stehen in der Politischen Ökologie daher die sozialen Beziehungen. Im Zusammenspiel mit strukturellen Faktoren bestimmen sie, wer die Fähigkeit besitzt, Kontrolle über den Zugang zu Land auszuüben, und wer Zugang zu Land nur indirekt erhält. Jesse Ribot und Nancy Peluso (2003) arbeiten strukturelle und relationale Mechanismen heraus, die zentral für den Zugang zu Land seien. Diese Mechanismen sind Zugang zu Technologie, Kapital, Arbeit, Märkten, Wissen, Behörden oder traditionellen Landrechtsinstitutionen und den $\mathrm{Zu}$ gang zu Land über soziale Zugehörigkeit. Konflikte, die im Zusammenhang mit land grabbing auftreten, sind aus dieser Perspektive Ausdruck der Aushandlung unterschiedlicher Formen von Zugang zu Land. Sie manifestieren sich in unterschiedlichen Formen des Widerstands gegen Enteignung und Vertreibung (Borras/Franco 2013; Engels/Dietz 2011), in Protesten gegen Aus- 
beutung, in Forderungen nach Integration und Teilhabe in Form von Arbeitsplätzen (Edelman et al. 2013; Li 2011; Oya 2013) sowie nach Partizipation und Mitbestimmung (Borras/Franco 2013) und in Widerstand gegen die Zerstörung von livelihoods (Escobar 2006). Neben Fragen der Ausgestaltung und Umsetzung agrarindustrieller Projekte werden in Konflikten im Zusammenhang mit land grabbing oftmals auch gegensätzliche Weltanschauungen und Naturvorstellungen sowie übergeordnete Forderungen nach gesellschaftlichem Wandel, sozialer Teilhabe und kultureller Anerkennung verhandelt. Diese betreffen häufig die Ausgestaltung von Landrechten, aber auch die Herausforderung dominanter Entwicklungsmodelle oder neoliberaler Wirtschaftspolitik, z.B. über die Verteidigung kleinbäuerlicher Landwirtschaft gegenüber der Ausweitung einer globalisierten agrarindustriellen Produktion (Dietz/Engels 2017).

Ebenso wie die zentralen Konfliktthemen unterscheiden sich Konflikte im Zusammenhang mit land grabbing auch hinsichtlich der beteiligten Akteure und der auftretenden Akteurskonstellationen. Anhand einer Auswahl an Studien bislang erforschter Konflikte, die im Zusammenhang mit land grabbing auftraten, schlagen Saturnino Borras und Jennifer Franco (2013) eine erste Typologie der involvierten Konfliktakteure und häufig auftretenden Akteurskonstellationen vor. Sie identifizieren drei Obergruppen von Konfliktakteuren, die typisch für Konflikte im Zusammenhang mit land grabbing sind: poor people, Unternehmen und staatliche Akteure. Traditionelle Autoritäten berücksichtigen sie nicht als Konfliktakteure.

Mit der Bezeichnung poor people knüpfen sie an den von Joan MartínezAlier (2002) geprägten Ausdruck environmentalism of the poor an. Dieser bezieht sich auf Konflikte, in denen marginalisierte indigene oder ethnische Gemeinschaften ihren Zugang zu natürlichen Ressourcen verteidigen. In der Regel forderten sie in diesen Konflikten kapitalistische Wirtschaftsmodelle heraus, die in einem Gegensatz zu den Lebensrealitäten vor Ort stünden (Escobar 1996; 2006: 7). Die meisten Studien zu land grabbing und Konflikten widmen sich in ihrer Analyse ebenfalls marginalisierten Gruppen von Landnutzer*innen in Ländern des Globalen Südens, die in den meisten Fällen ihr Land an ausländische Investoren verlieren. Andere Studien zeigen, dass das Phänomen nicht auf den Globalen Süden beschränkt ist und auch die Investoren differenziert betrachtet werden müssen (Brunner 2019; Franco/Borras 2013; van der Ploeg et al. 2015). Darüber hinaus weisen Borras und Franco (2013), wie auch viele andere aktuelle Beiträge (u.a. Boamah 2014a: 407; Gilfoy 2014; Hall et al. 2015; Ubink 2008a: 127), auf die Relevanz der sozialen Differenzie- 
rung der von land grabbing betroffenen Menschen hin. Mit der Verwendung der Bezeichnung poor people suggerieren sie jedoch gleichzeitig eine gewisse Homogenität der Gruppe, die gleichermaßen Kleinbäuerinnen und -bauern, Vertragslandwirt*innen, lokale Eliten, Kleinunternehmer*innen, traditionelle Autoritäten etc. umfassen kann. Unter Berücksichtigung dessen und in Abgrenzung dazu verwende ich in meiner Arbeit anstelle von poor people den Begriff Landnutzer*innen.

Laut Borras und Franco (2013: 1726) treten Konflikte im Zusammenhang mit land grabbing in den folgenden drei Akteurskonstellationen auf: zwischen Landnutzer*innen und Unternehmensakteuren, zwischen Landnutzer*innen und staatlichen Akteuren sowie zwischen unterschiedlichen Landnutzer*innengruppen. Der Fokus ihrer Analyse liegt auf den von land grabbing betroffenen Menschen und ihren jeweiligen Reaktionen. Dementsprechend vernachlässigen sie Konflikte zwischen Unternehmensakteuren und staatlichen Akteuren sowie zwischen unterschiedlichen Behörden. Insbesondere Letztere finden bislang in der Forschung zu land grabbing und Konflikten noch unzureichend Beachtung (Schenk 2018), ebenso wie Konflikte zwischen traditionellen Autoritäten und Landnutzer*innen, die im Zusammenhang mit land grabbing auftreten (Ausnahmen sind Campion/Acheampong 2014; Capps/Mnwana 2015; Mnwana 2019). Den von Borras und Fraco aufgeführten Akteurskonstellationen lassen sich ihrer Analyse entsprechend typische Konfliktgegenstände zuordnen.

Gegenstände von Konflikten zwischen Landnutzer*innen und Unternehmensakteuren seien hauptsächlich solche, die an anderer Stelle als Konflikte um Ausbeutung gefasst würden (vgl. hierzu Edelman et al. 2013). In diesen Konflikten gehe es insbesondere um die Ausgestaltung von Arbeitsbedingungen und die Verbesserung vertraglicher Vereinbarungen. Aber auch die Integration vorheriger Landnutzer*innen durch die Vergabe von Arbeitsplätzen oder im Rahmen von Vertragslandwirtschaft spiele eine Rolle. Zunehmend rückten zudem Umweltthemen wie z.B. die Verschmutzung von Wasser und Boden durch den Gebrauch von Chemikalien und damit zusammenhängende Gesundheitsaspekte in den Fokus (Borras/Franco 2013: 1728).

Konflikte zwischen Landnutzer*innen und staatlichen Akteuren seien vor allem solche, die an anderer Stelle als Konflikte um Enteignung gefasst würden (vgl. hierzu Edelman et al. 2013). Zentrale Themen in diesen Konflikten sind die Vertreibung von Kleinbäuerinnen und -bauern von ihrem Land und damit einhergehenden Umsiedlungsmaßnahmen oder Entschädigungszahlungen. Empirische Beispiele unterstreichen die Erkenntnis, dass der Ver- 
lust von Agrar- und Gemeindeland durch Vertreibung oder Enteignung zentraler Gegenstand in Konflikten im Kontext von land grabbing sei (Campion/Acheampong 2014: 6339; Edelman et al. 2013: 1527; White et al. 2012: 629). In vielen Fällen seien diesbezüglich jedoch nicht der Staat, sondern die Unternehmen für die Umsiedlung zuständig und Konflikte verliefen entsprechend zwischen Unternehmensakteuren und Landnutzer*innen (Drechsel et al. 2019).

In Konflikten zwischen unterschiedlichen Nutzer*innengruppen sind oftmals die unterschiedliche Betroffenheit lokaler Akteure und die damit einhergehenden Haltungen gegenüber land grabbing von Bedeutung. Nicht selten könne innerhalb einer Gemeinschaft beobachtet werden, dass einige Landnutzer*innen sich für und andere gegen ein bestimmtes Projekt aussprechen (Borras/Franco 2013: 1728). Die zentrale Bedeutung sozialer Differenzierung in der Analyse von Konflikten unterstreicht auch Pauline Peters (2004, 2013a, 2013b). In ihren Arbeiten führt sie die vermehrt auftretenden sozialen Konflikte um Land auf einen strukturellen Wandel der Gesellschaft und insbesondere eine zunehmende Klassenbildung zurück. Eine Anzahl an empirische Studien untermauern diese Erkenntnisse. Anhand einer Fallstudie zu einem agrarindustriellen Reisanbauprojekt in Mali zeigt Nicolette Larder (2015), dass betroffene Landnutzer*innen sowohl Allianzen für als auch Allianzen gegen das Projekt eingehen. Larder argumentiert, dass insbesondere Landnutzer*innen, die sich vom Staat im Stich gelassen fühlen und die abgesehen von ihrem Land keine alternative Einkommensquelle haben, agrarindustriellen Projekte gegenüber häufig positiv eingestellt sind. Sie sähen ihre Integration in diese Projekte als einzige Möglichkeit ihren Lebensstandard zu verbessern. Frankline Ndi und Simon Batterbury (2017) unterstreichen mit ihrer Fallstudie zu einem großflächigen Palmölprojekt in Kamerun, dass lokale Landnutzer*innen keinesfalls eine homogene Gruppe bilden. Mit ihrer Forschung zeigen sie, dass benachbarte Gruppen von Landnutzer*innen in Kontexten großflächiger Landumnutzung um die Sicherung des Zugangs zu Land konkurrieren und die Implementierung des agrarindustriellen Projekts daher zu Konflikten um Land zwischen unterschiedlichen Gemeinschaften führe. In diesen Konflikten handelten benachbarte Gemeinden die Ausübung von Kontrolle über zuvor gemeinschaftlich genutztes Land aus, mit der Aussicht es dem Unternehmen gegen Abgaben zur Verfügung stellen zu können. Eine ähnliche Beobachtung machen auch Burnod et al. (2013). Sie argumentieren, dass die Neuverhandlung vormals unklarer oder flexibler Grenzen und Zuständigkeiten in der Verwaltung von Land in Konflikten, die im Zusammenhang mit 
land grabbing auftreten von zentraler Bedeutung sei. In dem von Ndi und Batterbury (2017) untersuchten Fall, seien neben intergemeinschaftlichen Konflikten aber auch solche zwischen Landnutzer*innen und Unternehmen sowie Landnutzer*innen und lokalen Eliten, der nationalen Regierung oder anderen Unterstützer*innen der Unternehmen von Relevanz. Diese mehrfache Form der Verteidigung des Zugangs zu Land - einerseits gegen das Unternehmen und andererseits gegen andere Dörfer - spiegele die Heterogenität und soziale Differenzierung der Landnutzer*innen wider. Für die Ausgestaltung von Konflikten sei insbesondere die Vorherrschaft lokaler Eliten und damit im Umkehrschluss auch der Ausschluss lokaler Gemeinschaften in Verhandlungen großflächiger Landtransaktionen zu berücksichtigen. In dem von ihnen untersuchten Fall handelten lokale Eliten - zu denen sie auch traditionelle Autoritäten zählen - nach Eigeninteressen und nicht, wie es ihre Aufgabe gewesen wäre, im Interesse der Gemeinschaft (Ndi/Batterbury 2017: 47). Zu dieser Erkenntnis kommen auch empirische Studien aus anderen Ländern (Ahmed et al. 2018; Bartels et al. 2018; Nolte 2013). Der daraus resultierende Vertrauensverlust der lokalen Bevölkerung in traditionelle Autoritäten führe laut Ndi und Batterbury (2017) dazu, dass traditionelle Konfliktlösungsansätze seltener angewandt und stattdessen der Weg über das staatliche Rechtssystem in Anspruch genommen würde.

Konflikte zwischen unterschiedlichen Landnutzer*innen entstünden, wie andere Autor*innen betonen, zudem aus einer neuen Konkurrenz zwischen unterschiedlichen Gruppen von Landnutzer*innen. Durch den Wegfall von Gemeindeland, das vormals für vielfältige Zwecke genutzt wurde, seien Nutzer*innen gezwungen, auf andere Flächen auszuweichen und träten dadurch in Konkurrenz zu anderen Nutzer*innen. Ein prominentes Beispiel dafür sind Konflikte zwischen Landwirt"innen und Tierhalter"innen. Erstere nutzen das Land zum Anbau von Nahrungsmitteln, während Letztere es als Weidefläche für ihre Tiere benötigen, womit oftmals Schäden an der Ernte einhergingen (De Schutter 2011: 257).

Im Zusammenhang damit steht die Vergabe von »Ödland« (wastelands) (Cotula/Vermeulen 2009: 1241) - an anderer Stelle auch als »ungenutztes«, »leeres« oder »Grenzland (underutilized, empty, marginal land) bezeichnet (Peters 2013a: 15) - auf die zahlreiche Beiträge kritisch hinweisen. In der Regel handelt es sich dabei um Gemeindeland, für das niemand formalisierte Besitzrechte vorweisen kann und das staatliche Akteure gegenüber Investoren daher oft als ungenutzt bezeichnen. Jedoch ist Land, auf dem agrarindustrielle Projekte umgesetzt werden, fast nie »leer« oder »ungenutzt«. Denn 
soziale Gruppen nutzen Land auf unterschiedliche Weise, wie eine Vielzahl an empirischen Studien belegt (Borras/Franco 2013; Daley 2011; Murphy et al. 2017: 681f.). Diese beschreiben Land als die Grundlage vielfältiger ökonomischer Tätigkeiten wie kleinbäuerlicher Landwirtschaft, Wanderweidewirtschaft, Forstwirtschaft und dem Sammeln von Feuerholz, medizinischen Kräutern, Pflanzen und Früchten.

Vor allem letztere Tätigkeit, die hauptsächlich Frauen ausüben, fände bei der Umsetzung agrarindustrieller Projekte selten Berücksichtigung (Doss et al. 2014). Auch Katja Behrman et al. (2012) machen auf die besondere Betroffenheit von Frauen in diesem Kontext aufmerksam. Konflikte entstünden unter anderem in Zusammenhang mit der Exklusion von Frauen in Konsultierungsverfahren, durch ungleichen Zugang zu und ungleiche Kontrolle über Land von Männern und Frauen oder dadurch, dass geschlechter-spezifische Formen der Landnutzung nicht ausreichend berücksichtigt würden. Ebenfalls konfliktiv seien Szenarien, in denen nur Sektoren gefördert würden, in denen hauptsächlich Frauen arbeiten. Dadurch entstehe ein Ungleichgewicht in der Verteilung von Gewinnen, das zu innergemeinschaftlichen Konflikten führen könne. Auch wenn sich Geschlecht als eine wichtige Analysekategorie in Konflikten, die im Zusammenhang mit land grabbing stehen, erwiesen hat, haben damit zusammenhängende Aspekte in der bisherigen Forschung kaum Aufmerksamkeit erfahren (Borras/Franco 2013). Darüber hinaus sei Land nicht nur die Grundlage für die Ausübung wirtschaftlicher Tätigkeiten, sondern habe in vielen Gemeinschaften auch eine wichtige kulturelle Bedeutung. Es diene unter anderem der Durchführung kultureller und spiritueller Rituale, könne Ruhestätte der Ahnen oder heiliges Land sein (Borras/Franco 2013: 1726f.). Sein Wert gehe weit über den rein ökonomischen hinaus (Escobar 2006).

Die herausgearbeiteten Akteurskonstellationen treten in Konflikten im Kontext von land grabbing selten in einzelner Form auf. Fast immer überlappen sich unterschiedliche Konflikte oder treten parallel auf. So kämpfen in einem Fall Landnutzer*innen ohne formelle Eigentumsrechte vielleicht um Fragen der Teilhabe oder Entschädigung, während Vertragslandwirt*innen um die Ausgestaltung der Arbeitsbedingungen und Landeigentümer*innen mit entsprechenden formellen Eigentumsrechten gegen Enteignung kämpfen. Am häufigsten zu beobachten seien, so Hall et al. (2015: 471), Konflikte zwischen Landnutzer*innen und Unternehmen in Verbindung mit solchen zwischen Landnutzer*innen und dem Staat. 
Neben einer Vielzahl kritischer Beiträge zu Konflikten, die im Zusammenhang mit land grabbing stehen, existieren vermehrt auch solche, die sich mit möglichen Potenzialen großflächiger Landumnutzung befassen. Sie beziehen sich, wie Festus Boamah (2014b: 327) es fasst, auf einen Diskurs des development optimism. Das bedeutet, dass sie die inhärenten Gefahren von land grabbing anerkennen, sie jedoch vor allem auf mangelnde Transparenz und unvorteilhafte vertragliche Regelungen zurückführen. Sie setzen Konflikte, die im Zusammenhang mit land grabbing auftreten nicht in Bezug zu übergeordneten Gegenständen wie Enteignung oder Ausbeutung, sondern betonen hauptsächlich das Problem der negativen Auswirkungen. Sie beziehen sich vor allem auf Höhe und Auszahlungsmodi von Entschädigungszahlungen, Partizipation und Mitbestimmung in der Ausgestaltung der Verhandlungen und Umsetzung agrarindustrieller Projekte sowie das Fehlen, den Inhalt und die Umsetzung von Vereinbarungen zur sozialen Verantwortung von Unternehmen (Corporate Social Responsibility) (Campion/Acheampong 2014: 6339, 6341; Cotula/Berger 2017; Cotula et al. 2014). Darüber hinaus richten zahlreiche Beiträge ihre Aufmerksamkeit in diesem Zusammenhang auf den fehlenden Informationsfluss und die mangelnde Einbindung betroffener Nutzer*innengruppen von Seiten der Unternehmen, des Staats sowie traditioneller Autoritäten (Cotula et al. 2014: 918; Peters 2013a: 16). Diese Studien hinterfragen land grabbing also nicht per se. Zudem vernachlässigen sie weitgehend die Berücksichtigung zugrundeliegender ungleicher Machtverhältnisse und rücken vielmehr das Entwicklungspotenzial agrarindustrieller Projekte in den Vordergrund. Sie argumentieren, dass dieses die negativen Auswirkungen von land grabbing abschwächen könne.

\subsection{Konflikte um Land und die Bedeutung sozialer Zugehörigkeit}

Konflikte um Land treten nicht erst seit dem Anstieg von land grabbing in den frühen 2000er Jahren auf. Vielmehr ist Land seit jeher ein umkämpftes Gut, und Konflikte um dieses Gut seien, so Sara Berry, nicht allein über knapper werdende Ressourcen oder eine zunehmende Kommodifizierung von Land zu erklären (1992). In vielen Fällen seien sie Ausdruck für die Neuverhandlung von Rechten, die den Zugang zu, die Nutzung von und die Kontrolle über Land bestimmen (Kirst/Engels 2014). Zahlreiche Autor*innen verweisen darauf, dass diese Rechte oftmals in einem wechselseitig konstitutiven Verhältnis zu sozialer Zugehörigkeit, Macht und Herrschaft stünden (Berry 2009b; 
Greco 2015; Lund 2011; Lund/Boone 2013; Sikor/Lund 2009a). Die kommerzielle Nutzung von Land könne bestehende Rechte an Land überlagern, ersetze sie in der alltäglichen Praxis der Landnutzer*innen jedoch nicht (Berry 2017: 109). Für die Analyse von Konflikten um Land seien daher Kategorien sozialer Zugehörigkeit von zentraler Bedeutung. Dies gelte insbesondere für Kontexte, in denen Gewohnheitsrecht oder traditionelle Landrechtsinstitutionen den Zugang zu, die Nutzung von und die Kontrolle über Land bestimmen, wie es in den meisten Ländern Subsahara-Afrikas der Fall ist. Denn in traditionellen Landverwaltungssystemen können verschiedene Landnutzer*innen unter Bezug auf mündlich übermittelte Normen und Regeln vielfache, teils parallel existierende Rechte an Land einfordern. Innerhalb der landbesitzenden Gruppe sind, je nach Status und Position, unterschiedliche Personen für die Vergabe dieser Rechte zuständig (Berry 1993). Die Fähigkeit, den Zugang $\mathrm{zu}$ Land $\mathrm{zu}$ kontrollieren oder bestimmte Rechte an Land einzufordern, ist in der Regel eng mit der vermeintlichen Herkunft einer Person oder ihrer Zugehörigkeit zu einer bestimmten (ethnischen) Gruppe verknüpft. Bis heute bestimmen das Erbe der Kolonialpolitik und deren Einfluss auf die Bedingungen, die den Zugang zu Land strukturieren, maßgeblich die Ausgestaltung traditioneller Landverwaltungssysteme (Berry 1992).

\section{Die koloniale Interpretation traditioneller Landrechte}

Wie verschiedene Autor*innen zeigen, war die Ausübung von Kontrolle über Land ein zentrales Anliegen aller Kolonialregime (Amanor 1999; Berry 1992; Peters 2013b). Ziel der Kolonialregime sei es gewesen, anhand der Kontrolle über Land politische Macht über die auf dem Land lebenden Personen zu erlangen und aufrechtzuerhalten sowie gleichzeitig Gewinne aus der kommerziellen Nutzung des Landes zu ziehen. Die Gewinne aus der kommerziellen Nutzung des Landes seien jedoch gleichermaßen ein Anreiz für die Kolonialmacht wie auch für die Kolonialisierten gewesen. Dadurch sei eine neue Konkurrenz um Land und damit zusammenhängende Einkünfte durch cash crops oder Lohnarbeit entstanden (Berry 1992). Da die Kolonialregierung einen direkten Zusammenhang zwischen der Ausübung politischer Macht und territorialer Kontrolle sah, strebten sie die Formalisierung traditioneller Landund Nutzungsrechte an. Diese sollte die Kontrolle des Landzugangs lokaler Gemeinschaften erleichtern.

Um unterschiedliche territoriale Gebiete voneinander abzugrenzen und kontrollierbar zu machen, verzeichneten die Kolonialregime zunächst die 
vermeintlichen territorialen Zuständigkeitsbereiche traditioneller Autoritäten auf Landkarten. Gleichzeitig strebten sie eine enge Zusammenarbeit mit lokalen Eliten und chiefs an. Diese brachten den Kolonialbeamt*innen den Inhalt traditionellen Rechts näher und waren später für die Durchsetzung des formalisierten traditionellen Rechts zuständig. Im System der indirekten Herrschaft übertrugen die Kolonialregierungen loyalen, teilweise neu eingesetzten chiefs die Verwaltung der neu geschaffenen Territorien und stärkten auf diese Weise die Verbindung zwischen politischer Macht und Kontrolle über den Zugang zu Land. chiefs hatten nicht nur das Recht Statute $\mathrm{zu}$ erlassen, mithilfe derer sie über Personen in ihrem Gebiet herrschten, sondern sie führten diese Gesetze auch aus und nahmen darüber hinaus die Rolle von Verwaltungsbeamten ein. Des Weiteren waren sie für die Schlichtung jeglicher Konflikte zuständig. Die Amtsgewalt der chiefs vereinigte also in einer einzigen Person judikative, legislative, exekutive und administrative Macht (Mamdani 1996: 23). Diese war jedoch nicht gänzlich unabhängig vom zentralen Kolonialstaat. Kooperationsunwillige oder widerständige chiefs enthob die Kolonialmacht (teilweise gewaltsam) ihres Amts (von Trotha 1996: 80). In diesem Zuge habe sich durch die Erfindung neuer sozialer Kategorien die Kontrolle über Land zugunsten derjenigen verschoben, die in der Hierarchie des traditionellen Herrschaftssystems weiter oben stehen, wie Pauline Peters (2013a: 4) zeigt: von Familienoberhäuptern, Älteren einer Abstammungslinie und village chiefs, hin zu paramount oder territorial chiefs. Hierarchien seien entweder neu geschaffen oder zumindest verstärkt worden. Dadurch verstärkte sich nicht zuletzt auch die Konkurrenz unterschiedlicher traditioneller Autoritäten - insbesondere um Land.

Unter direkter Herrschaft, wie in französischen Kolonien, übernahm offiziell die Kolonialregierung die Landverwaltung. Dieser Anspruch existierte jedoch meist nur in der Theorie, da die Kapazitäten für die Durchsetzung fehlten (Berry 2017: 108). In der Praxis hingegen bestanden traditionelle Landverwaltungssysteme sowohl unter indirekter als auch unter direkter Herrschaft fort, wobei die koloniale Praxis in beiden Fällen einen Einfluss auf ihre Ausgestaltung ausübte. Wie Richard Kuba und Carola Lentz (2003) für Burkina Faso zeigen, erfand die Kolonialregierung unter direkter Herrschaft zwar keine gänzlich neuen sozialen Kategorien, wie es unter indirekter Herrschaft der Fall war, institutionalisierte jedoch ethnische Kategorien als Legitimationselement für politischen Status und politisierte diese durch ihre Festschreibung in räumlich begrenzte Zuständigkeitsbereiche. Indem die Kolonialregierung durch traditionelle Autoritäten verwaltetes Land als ein durch eth- 
nische Zugehörigkeit bestimmtes Territorium fasste, habe sie eine Hierarchie des Landzugangs auf der Landkarte eingezeichnet. Wie auch Mahmood Mamdani (2001) verdeutlicht, betonte die Definition politischer und territorialer Macht mittels festgeschriebener geographischer Grenzen die Bedeutung von Gruppenzugehörigkeit für die Einforderung von Rechten an Land. Denn über die Grenzziehung definierte die Kolonialregierung auch, wer in diesem Gebiet die »Einheimischen « waren, die entsprechend Rechte an Land geltend machen konnten, und wer die »Fremden« waren, deren Zugang zu Land von der Zustimmung der jeweiligen traditionellen Autorität abhing. In der vorkolonialen Praxis seien soziale Grenzen jedoch tendenziell fließend, durchlässig und - anders als die Kolonialbeamt*innen annahmen - nicht deckungsgleich mit territorialen Grenzen gewesen. Der Verlauf historisch gewachsener territorialer Grenzen, die durch unterschiedliche Personengruppen genutztes oder bewohntes Land unterteilten, sei selten klar definiert gewesen. Darüber hinaus fiel die Fähigkeit, Kontrolle über Land ausüben zu können, nicht immer zusammen mit der Herrschaft über alle Personen, die dieses Land nutzten. Das habe die Einteilung in Einheimische und Fremde problematisch gemacht. Je nachdem in welcher vorherigen Beziehung neue Migrant*innen zu Mitgliedern einer Gemeinschaft standen, seien sie bei ihrer Ankunft nicht zwangsläufig als Fremde eingestuft worden. Gesellschaftliche Positionen konnten zudem über die Zeit neu verhandelt werden, was eine feste Einteilung in die eine oder andere Kategorie erschwert habe (Berry 2002: 643ff.).

Die Formalisierung von Land- und Nutzungsrechten erfolgte über die Kodifizierung derjenigen Regeln und Normen in Kolonialrecht, welche die Kolonialregierung als traditionelles Gewohnheitsrecht anerkannte. Gewohnheit sei jedoch, wie Sara Berry (2002: 643) veranschaulicht, ein sich wandelndes Kaleidoskop von Erzählungen und Interessen gewesen. Erzählungen über die Vergangenheit seien vielfältig und oftmals widersprüchlich gewesen. Lokale Regeln, Normen, Gesetze und Bräuche unterschieden sich maßgeblich in $\mathrm{Ab}$ hängigkeit davon, welche Person mit welchen Eigeninteressen Auskunft darüber gab.

Die unterschiedliche Darstellung traditioneller Landrechte sei nicht zuletzt Ausdruck bestehender Kämpfe und Aushandlungsprozesse um die Kontrolle von Land und damit zusammenhängender Macht gewesen (Berry 1992: 333). Durch die Kodifizierung vermeintlich traditioneller, vor allem aber auch widersprüchlicher Regeln und Normen in koloniales Recht habe die Kolonialregierung ein Landrechtssystem erschaffen, das auf bestehen- 
den Konflikten um Macht und sozialer Zugehörigkeit aufbaute. Indem die Kolonialmächte Zugangsrechte $\mathrm{zu}$ Land in direkten Bezug zu Kategorien sozialer Zugehörigkeit setzten, sei diese zum Gegenstand kontinuierlicher Aushandlungsprozesse geworden, um wirtschaftliche und politische Gelegenheiten möglichst zum eigenen Vorteil nutzen zu können. Das Ergebnis seitens der Landnutzer*innen sei eine andauernde Aushandlung darüber gewesen, welchen Einfluss der Zugang zu Land auf die Zuschreibung sozialer Zugehörigkeit habe und wie im Gegenzug soziale Zugehörigkeit den Zugang zu Land bestimme (Berry 1992: 346). Martin Chanock (1991) argumentiert diesbezüglich, traditionelle Landrechte seien während der Kolonialzeit in einem Dialog zwischen Herrschenden und Beherrschten entstanden. Die Kolonialregierung habe durch die »Erfindung« traditioneller Landrechte die Entstehung von individuellem Landbesitz verhindert. Gleichzeitig stand sie Bestrebungen junger Menschen, Land der alleinigen Kontrolle älterer Männer zu entziehen, ambivalent gegenüber. Das kontinuierliche Streben der Kolonialmächte, die richtige, also für sie vorteilhafteste Version von Gewohnheitsrecht zu etablieren, habe bewusst hegemoniales, in der Praxis jedoch instabiles Wissen produziert. Kolonialmächte erachteten Tradition und Gewohnheit als etwas Zeitloses. chiefs und Dorfälteste, die vorgaben vermeintliche Traditionen $\mathrm{zu}$ kennen, erzählten jedoch widersprüchliche Geschichten. Gewohnheit selbst sei dadurch zu einer scheinbar festen Menge an Grundsätzen geworden, die jedoch durch andauernde geschichtliche Interpretation und Aushandlung gleichzeitig Gegenstand kontinuierlicher Veränderung gewesen seien (Berry 2002: 645). Die indirekte Herrschaft der Kolonialregime, habe es weder ermöglicht ein neues Rechtssystem zu erschaffen, noch das traditionelle zu erhalten oder ihre erfundene Version »traditioneller« afrikanischer Gesetze zu etablieren. Aufgrund ungenügender finanzieller Mittel und Durchsetzungskraft sowie fehlender direkter Kontrolle bestimmten die neuerfundenen »traditionellen « Gesetze die koloniale Gesellschaftsordnung nur in der Theorie. In der Praxis führten sie zu Auseinandersetzungen um die Bedeutung von Land für politische Macht und um die Anerkennung konkurrierender Versionen vermeintlicher Tradition. Dies habe wiederum einen Einfluss auf Kämpfe um Herrschaft und um Zugang zu Ressourcen gehabt. Durch die Anerkennung konkurrierender Erzählungen und damit verbundenen Ansprüchen auf territoriale Kontrolle habe die indirekte Kolonialherrschaft die Instabilität der Bedingungen, die den Zugang zu Land bestimmten, gefördert und lokale Herrschaftsstrukturen fragmentiert. 


\section{Traditionelle Landrechtssysteme im Kontext aktueller Entwicklungen}

Was wir heute als Gewohnheitsrecht bezeichnen, ist also nicht der Fortbestand prä-kolonialer Normen, sondern ein Rechtssystem, das auf der kolonialen Interpretation von »Tradition« beruht (vgl. Ranger 1983). Wie Philippe Lavigne Delville (2000: 99) es fasst, gebe es keine »traditionellen« oder gewohnheitsrechtlichen Landnutzungssysteme an sich, sondern nur Landverwaltungsformen, die auf gewohnheitsrechtlichen Prinzipien beruhen. Unter Berücksichtigung historischer Entwicklungen sprechen zahlreiche Autor*innen daher heute von »neo«-traditionellen Landrechtssystemen oder auch "neo«-traditionellen Autoritäten (vgl. Boone 2015; Knierzinger 2011; Lund/Boone 2013). Insbesondere in Bezug auf die jüngere Vergangenheit besteht in der Literatur Konsens, dass sich traditionelle Landrechtssysteme über die Zeit verändern und mit Maßnahmen wie Dezentralisierungsund Strukturanpassungsprogrammen, der Liberalisierung der Märkte, wirtschaftlichem Wachstum und zunehmender agrarindustrieller Nutzung interagieren (vgl. Juul/Lund 2002b; Lentz 2010; Toulmin/Quan 2000; Ubink 2008b). Berry (2017) veranschaulicht dies in ihrem Beitrag zu Veränderungen in der Landpolitik und den Praktiken der Landvergabe seit den 1990er Jahren, die sie an Beispielen aus Ghana und der Elfenbeinküste illustriert. Sie argumentiert, traditionelle Landrechte würden insbesondere dann neu verhandelt, wenn der Bodenwert ansteige (vgl. auch Amanor 1999). Als Beispiel dafür nennt sie die in den 1990er Jahren verbreiteten Bestrebungen, in Ländern des Globalen Südens die Registrierung individueller Landrechte $\mathrm{zu}$ fördern. Durch diese Strategie versuchten nationale Regierungen in Kooperation mit internationalen Entwicklungsorganisationen wie der Weltbank, Eigentumsrechte zu stärken, um auf diese Weise Investitionen in Land anzuregen. Dadurch schrieben sie Land einen neuen kommerziellen Wert zu. In der Praxis habe das dazu geführt, dass Landnutzer*innen neue Ansprüche auf Land stellten oder alte wiederbelebten, um individuelle Landnutzungsrechte an zuvor gemeinschaftlich geteiltem Land zu erhalten.

Die flexible Ausgestaltung von Landrechten habe es in diesem Zusammenhang erlaubt, neu zu definieren, wer unter welchen Bedingungen und auf welcher Grundlage Zugang zu Land erhält und wer in Konfliktfällen entscheiden kann, wessen Rechte als legitim gelten. Diese viel diskutierte Flexibilität und Verhandelbarkeit, die als typisch für Landzugangs- und Nutzungssysteme in Subsahara-Afrika gelten, stellt Peters (2004) infrage. Sie argumentiert, dass allgegenwärtige Konkurrenz und Konflikte um Land auf 
dem afrikanischen Kontinent die Darstellung traditioneller Landrechtssysteme als offen, verhandelbar und anpassungsfähig in Zweifel zögen. Stattdessen führten sie zu einer zunehmenden Exklusion, gesellschaftlicher Spaltung und Klassenbildung. Anstatt von einer allgemeinen Flexibilität und Verhandelbarkeit auszugehen, sei es nötig zu analysieren, wem traditionelle Landrechtsinstitutionen zugutekämen und für wen sie sich nachteilig auswirkten. Dies ist besonders relevant vor dem Hintergrund, dass es seit den frühen 200oer Jahren im Rahmen von Landreformen verstärkte Bestrebungen gibt, traditionelle oder Gewohnheitsrechte offiziell anzuerkennen und in nationales Recht zu integrieren (Toulmin/Quan 2000). Die Betonung der Verhandelbarkeit und Mehrdeutigkeit traditioneller Landrechtssysteme habe zu einer Zunahme von Studien geführt, die sich auf deren Bedeutung für Handlungsmacht und gesellschaftlichen Gestaltungsspielraum konzentrierten. So geriete aus dem Blickfeld, wer die Gewinnenden und Verlierenden seien. Wichtig sei, präzise zu analysieren, welche sozialen und politischen Verhältnisse Landzugang und -nutzung bestimmen, insbesondere mit Hinblick auf Ungleichheit. Die Analyse müsse, unter Rückbezug auf Kategorien wie Ethnizität, Klasse, Geschlecht und Alter berücksichtigen, wer mit welchen Auswirkungen in der Lage sei Landrechte zu verhandeln. Damit schließt Peters an Kojo Amanor (1999) an. Er argumentiert, die Hervorhebung der flexiblen Verhandelbarkeit traditioneller Landrechtssysteme führe dazu, den Einfluss Einzelner auf Debatten darüber, was Tradition ausmache und welche Regeln den Zugang zu Ressourcen bestimmen sollten, zu überschätzen. Seiner Ansicht nach seien nur die Reichen und Mächtigen, über Investitionen in soziale Netzwerke, in der Lage Letzteres festzulegen. Frauen, jungen Menschen und Migrant"innen seien in der Durchsetzung ihrer Forderungen tendenziell benachteiligt. Mit zunehmender Konkurrenz um Land käme es daher nicht nur vermehrt zu Konflikten zwischen Indigenen und Migrant*innen, sondern auch innerhalb von Familien, in denen häufig junge Menschen um die Beständigkeit ihres Zugangs zu Familienland bangten. Die Kommodifizierung von Land und die in diesem Zusammenhang entstehende Vernetzung nationaler Eliten, Staatsangestellter und internationaler Unternehmen durchdrängen Beziehungen zwischen Generationen, Verwandten und Geschlechtern und trügen zu gesellschaftlichen Verhältnissen bei, die durch soziale Ungleichheit gekennzeichnet seien (Amanor 2001).

Auch wenn es übergeordnete Entwicklungen gebe, die soziale Verhältnisse und Zugang zu Land in einen Zusammenhang setzten, sei die Ausgestaltung von Konflikten um Land und die Macht von Akteuren maßgeblich durch 
den jeweiligen Kontext bestimmt. Das verdeutlicht Berry (2017) beispielhaft anhand eines Vergleichs von Ghana und der Elfenbeinküste. Sie argumentiert, dass es zu berücksichtigen gelte, wie und in welchem Rahmen unterschiedliche aktuelle Machtstrukturen, politischer Wettstreit und verschiedene Formen des Landzugangs und der Landnutzung aufeinanderträfen. Während gegenwärtige Kämpfe um Land zu einem gewissen Grad das Erbe der Kolonialherrschaft widerspiegelten, habe sich die Ausgestaltung wirtschaftlicher Möglichkeiten, politischen Wettbewerbs und sozialer Differenzierung in verschiedenen Ländern individuell entwickelt und verändert. Die Ausgestaltung gegenwärtiger Konflikte um Land sei daher keinesfalls allein auf die gemeinsame koloniale Erfahrung indirekter Herrschaft zurückzuführen, sondern immer in Bezug zu aktuellen Entwicklungen zu sehen (Berry 2017: 115).

\section{Die Bedeutung von Macht für Zugang zu Land}

Bezüglich des Verhältnisses von sozialer Zugehörigkeit und Zugang zu Land in traditionellen Landverwaltungssystem widmen sich einige Autor*innen der Frage, wie sich Macht und Herrschaft sowie der Zugang zu und die Kontrolle über Land noch heute wechselseitig bedingen (Sikor/Lund 2009b). Der Zugang zu und der Besitz von Land, so argumentieren Thomas Sikor und Christian Lund (2009a), stünden in engem Zusammenhang mit Macht und Herrschaft. Denn um Zugang zu oder Besitz von Land zu sichern, bedürfe es einer gesellschaftlich legitimierten Institution, welche die Macht habe, diese Rechte anzuerkennen. Die Legitimität einer solchen Institution und die damit zusammenhängende Macht, resultierten unter anderem aus ihrer Anerkennung. Anerkennung zeige sich darüber, dass Individuen, die nach Zugang $\mathrm{zu}$ Land streben, bestimmten Institutionen die Kompetenz einräumten, für die Vergabe und Anerkennung von Landrechten zuständig zu sein.

Für die Frage, wer sich Land aneignen kann, wer es besitzen oder vergeben kann, seien Aspekte wie die Zugehörigkeit zu einer lokalen Gemeinschaft, Status und Zugehörigkeit relevant. In vielen Kontexten ist Autochthonie ein zentrales Konzept für die Legitimierung von Ansprüchen auf Land. Autochthonie bindet kollektive Identität an ein Territorium. Damit grenze dieses Konzept soziale Gruppen, die für sich beanspruchen »schon immer« oder zumindest »schon lange« an einem bestimmten Ort gelebt zu haben, von solchen $\mathrm{ab}$, die erst »später« hinzugekommen seien, was Konsequenzen für den Zugang zu Land habe (Kirst/Engels 2012). Zusätzlich dazu seien in 
den meisten Fällen soziale Kategorien wie Geschlecht, Alter, Klasse, race, Kaste und Ethnizität für die Einforderung von Landansprüchen relevant (Boone 2014; Lund/Boone 2013). Christian Lund (2011) verdeutlicht, dass soziale Zugehörigkeit und Status nicht automatisch Rechte nach sich ziehen, ihre Einforderung jedoch legitimieren können. Fehlende Zugehörigkeit zu einer lokalen Gemeinschaft könne es einer Person im Gegenzug erschweren, Rechte einzufordern. Einmal erworbene Rechte seien nicht notwendigerweise beständig, sondern in erster Linie vorübergehende Erfolge von Bemühungen, Ansprüche auf Land zu stärken und festzuschreiben. Sie stünden im Wettstreit mit anderen Versuchen, sie bloßzustellen oder zu ersetzen (Moore 1978). Ebenso seien auch Gruppengrenzen nicht endgültig definiert, sondern durchlässig. Das ermögliche es Außenstehenden, die Zugehörigkeit zu einer Gruppe und mit ihr auch Ansprüche auf Land zu erlangen. Außenstehende können Gruppenzugehörigkeit beispielsweise über Heirat und damit verbundene Brautgeschenke, über besondere Loyalität gegenüber oder besonderes Engagement in der Gemeinschaft erwerben (Berry 1989; Kirst/Engels 2012).

In Konflikten um Land werde soziale Zugehörigkeit beispielsweise über die engere Definition von Gruppengrenzen neuverhandelt, wodurch sich der Kreis derjenigen, die Rechte an Land auf legitime Weise einfordern können, verkleinere (Peters 2004). Unterschiedliche Studien beschreiben dies für die Elfenbeinküste (Berry 2009b), Botswana (Peters 1994), Malawi (Kishindo 2004), Äthiopien (Feyissa 2005), Zimbabwe (Moore 2005), Kenia (Moore 1991), Südafrika (Capps/Mnwana 2015) und Ghana (Amanor 1999; Ubink 2008b). Konkurrierende Ansprüche auf Land würden in diesem Zusammenhang häufig über historische Präzedenzfälle gerechtfertigt, die relevant für bestehende Besitz- und Machtverhältnisse sind (Berry 2002). In seinem Beitrag $\mathrm{zu}$ Strategien der Legitimierung und Sicherung von Kontrolle über Land illustriert Lund (2013) diesbezüglich, wie unterschiedliche Erzählungen über die Vergangenheit konkurrierende Landansprüche in traditionellen Landrechtssystemen rechtfertigen. Die Kontrolle über Land sei eng an diskursive Strategien geknüpft. Insbesondere die Rekonstruktion der Vergangenheit sowie unterschiedliche Konzeptualisierungen von Raum - entweder als Besitz oder als Territorium - seien in diesem Zusammenhang zentral.

Erkenntnisse aus den Arbeiten $\mathrm{zu}$ Land, sozialer Zugehörigkeit und Konflikten unterstreichen die Bedeutung sozialer Zugehörigkeit. Für mein Forschungsvorhaben ergibt sich daraus die Notwendigkeit, multiple, parallel existierende oder sich überlappende Rechte an Land in der Analyse von Konflikten um Land zu berücksichtigen, da diese in traditionellen Land- 
rechtssystemen oftmals mit Kategorien sozialer Zugehörigkeit verschränkt sind. Je nach sozialer Zugehörigkeit können Individuen und Gruppen sich unterschiedlicher Mechanismen bedienen, über die sie Zugang zu Land erlangen können. Traditionelle Autoritäten kontrollieren einige dieser Mechanismen, jedoch nicht alle. Im Laufe der Zeit können sich diese Mechanismen verändern, da traditionelle Normen und Rechte nicht beständig, sondern verhandelbar und wandelbar sind, wenn auch nicht beliebig und durch jeden. Für die Auswertung meiner empirischen Daten bedeutet dies, präzise - und insbesondere unter Berücksichtigung sozialer Differenzierung - zu analysieren, welche sozialen und politischen Verhältnisse Zugang $\mathrm{zu}$ Land bestimmen und wer in Konflikten um Zugang die Gewinnenden und Verlierenden sind. Der Rückbezug auf Kategorien sozialer Differenzierung ermöglicht es zu erfassen, wer mit welchen Auswirkungen in der Lage ist, Rechte an Land aufrechtzuhalten oder neu zu verhandeln und wer nicht. Bislang findet die soziale Differenzierung lokaler Gemeinschaften in der Forschung zu Konflikten im Zusammenhang mit land grabbing noch unzureichend Berücksichtigung.

\subsection{Traditionelle Autoritäten als Intermediäre}

Um mich der Rolle traditioneller Autoritäten in Konflikten, die im Zusammenhang mit land grabbing auftreten, zu nähern, knüpfe ich in meiner Forschung an eine dritte Debatte an: diejenige zu traditionellen Autoritäten als Intermediäre.

Bereits während der Kolonialzeit, insbesondere unter indirekter Herrschaft, schrieben Kolonialverwalter*innen traditionellen Autoritäten eine vermittelnde Funktion zwischen der Bevölkerung und dem Kolonialstaat zu. Dies belegt eine umfangreiche Literatur aus der historischen Forschung (Crowder 1964; Eckert 1999, 2007; Eriksen 2001). Mit dem Ende der Kolonialzeit verloren traditionelle Autoritäten zunächst an Prominenz in der Politik. Als Intermediären sind sie erst in jüngerer Zeit wieder in den Fokus der Aufmerksamkeit gerückt. Insbesondere internationale NGOs, Geberund Entwicklungsorganisationen haben ihren Nutzen als Mediator*innen und Entwicklungsagent*innen entdeckt. Aktuelle Studien dazu beziehen sich auf unterschiedliche Kontexte, in denen traditionelle Autoritäten als Intermediäre zwischen verschiedenen Akteuren agieren und teilweise sogar bewusst als solche eingesetzt werden. Kritische Forschungen dazu verweisen 
auf eine mangelnde Berücksichtigung der historischen Entwicklung traditioneller Herrschaftssysteme, insbesondere mit Bezug auf die Kolonialzeit. Die fehlende Berücksichtigung der historischen Entwicklung berge die Gefahr, gesellschaftlich verankerte Ungleichheit zu reproduzieren.

\section{Traditionelle Autoritäten als Mediator*innen und Entwicklungsagent*innen}

Mit dem Ende der Kolonialzeit verloren traditionelle Autoritäten zunächst an Prominenz in der Politik. In den 1990er Jahren kam seitens internationaler Geber- und Entwicklungsorganisationen jedoch ein erneutes Interesse an der Kooperation mit traditionellen Autoritäten auf, das Ann Whitehead und Dzodzi Tsikata als »return to the customary« bezeichnen (2003: 68). Das erneute Interesse an der Zusammenarbeit mit traditionellen Autoritäten bezog sich zunächst auf die Bereiche Entwicklungsförderung, lokale Regierungsführung und Konfliktmanagement (Ubink 2008c). Später rückten traditionelle Autoritäten ebenfalls mit Blick auf die dezentrale Verwaltung natürlicher Ressourcen als günstige und kulturell legitime Akteure in den Fokus (Capps 2018). Das erneute Interesse an der Zusammenarbeit mit traditionellen Autoritäten entstand in einem Kontext der Demokratisierung, die mit zunehmender Kritik internationaler Geber- und Entwicklungsorganisationen an der Leistungsfähigkeit und institutionellen Qualität vieler afrikanischer Staaten einherging. Die neu gegründeten Staaten sahen sich mit dem gleichen Dilemma wie schon die Kolonialregierungen konfrontiert: die Verwaltung riesiger Gebiete unter Verfügung geringer Ressourcen und schwacher staatlicher Institutionen in ländlichen Gegenden bewältigen zu müssen (Baldwin 2016). Organisationen wie die Weltbank sahen einen direkten Zusammenhang zwischen der vermeintlich schlechten wirtschaftliche Entwicklung und der schwachen Leistung lokaler staatlicher Institutionen (Brautigam 1992; Hydén/Bratton 1992; World Bank 1989, 1992, 1994). Die Kooperation mit traditionellen Autoritäten sollte die Regierungsführung auf lokaler Ebene stärken und fehlende staatliche Kapazitäten ausgleichen (vgl. z.B. World Bank 2002). Als innovative Strategie fügte sie sich zudem nahtlos in das Bild einer auf der Partizipation der »lokalen Bevölkerung« beruhenden Entwicklungspolitik ein (OECD/DAC 1996: 9). Bei der Wahl traditioneller Autoritäten als Kooperationspartner*innen stand vor allem deren vermeintliches soziales Kapital im Vordergrund. Als kulturell versierte Repräsentant*innen ihrer Gemeinschaft verkörperten sie sozialen Zusammenhalt 
und gemeinschaftliches Handeln. Dadurch seien sie zu einem Sinnbild für eine erfolgreiche nachhaltige Entwicklung geworden, die aus Sicht vieler Entwicklungsorganisationen nur aus einer authentischen lokalen Kultur heraus entstehen könne (Grischow 2008: 64). Im Kontext zunehmender innerstaatlicher Konflikte und einem erhöhten Bedarf an neuen Konfliktlösungsansätzen, stieg parallel auch das Interesse an traditionellen Autoritäten als Mediator*innen in Konflikten. Befürworter*innen traditioneller Konfliktlösungsansätze betonen diesbezüglich die niedrigschwellige Zugänglichkeit traditioneller Institutionen. Sie argumentieren, dass traditionelle Rechtsprechung günstiger, schneller und leichter verständlich sei als der Weg über das staatliche Rechtssystem, das auf viele einen einschüchternden Effekt habe. Konfliktschlichtung sei auch deshalb ein zentraler Aufgabenbereich traditioneller Autoritäten, da diese auf stabilisierende und vereinende Art mit Konflikten und Streitfällen umgingen (Adjei et al. 2017: 314). Seit Beginn der 200oer Jahre beschäftigen sich zahlreiche Autor*innen mit den Möglichkeiten, Herausforderungen und Grenzen traditioneller Konfliktlösungsansätze sowie der Rolle traditioneller Autoritäten in der Bearbeitung von Konflikten. Neben wissenschaftlichen Studien (u.a. Buckley-Zistel 2005; Moe/Simojoki 2013; Rukuni et al. 2015; Santschi 2014; Tubiana et al. 2012; Zartman 2000) kommen Beiträge vermehrt auch aus der entwicklungs- und friedenspolitischen Praxis (Boege 2006; OECD 2001). Einige Autor*innen argumentieren, traditionelle Autoritäten, welche die Kolonialzeit überdauert hätten, verfügten über eine besondere Legitimität innerhalb ihrer Gemeinschaft. Während der Kolonialzeit seien sie häufig zentrale Figuren im antikolonialen Widerstand und damit Sinnbilder kultureller Identität gewesen. Ihre soziale und politische Legitimität leite sich daher auch heute noch aus prä-kolonialer Zeit ab (Ray/Reddy 2003).

\section{Kritische Perspektiven auf traditionelle Autoritäten als Intermediäre}

Argumentationsweisen wie diese rechtfertigten und stärkten die Funktion traditioneller Autoritäten als so genannte Entwicklungsagent*innen (agents of development) oder Entwicklungsvermittler*innen (development broker) (Grischow 2008) und legitimierten ihre zentrale Rolle in der Schlichtung von Konflikten. Sie blieben jedoch nicht unangefochten. Gleichwohl traditionelle Autoritäten im Zuge einer Welle der Demokratisierung als Repräsentant*innen lokaler Gemeinschaften wiederentdeckt wurden, weisen Kritiker"innen darauf hin, dass traditionelle Herrschaft demokratische Prinzipien gefährden kön- 
ne. Denn traditionelle Autoritäten werden in der Regel ernannt und nicht demokratisch gewählt. Gemeinschaften hätten daher kaum eine Möglichkeit, die Rechenschaftspflicht traditioneller Autoritäten einzufordern, so dass ihr Handeln oftmals intransparent sei. Daraus könne sich eine Gefährdung für Errungenschaften der Demokratie wie Gleichheit, Menschenrechte oder Geschlechtergleichstellung ergeben (Buur/Kyed 2007; Ubink 2008c). In Bezug auf die Nachteile traditioneller Konfliktbearbeitung verweist Volker Boege (2006) beispielsweise darauf, dass traditionelle Herrschaftssysteme immer auf gewisse Weise die "gute alte Ordnung« reproduzierten und anfällig für Missbrauch seien. Das könne sich als nachteilig für die schwachen Menschen der Gemeinschaft erweisen.

Insbesondere aus der politischen Anthropologie existiert eine Anzahl an kritischen Analysen, die sich mit traditionellen Autoritäten als Entwicklungsagent*innen oder Entwicklungsvermittler*innen befasst. Im Fokus der Kritik steht unter anderem der Social Capital Ansatz. Befürworter*innen des Social Capital Ansatzes definieren traditionelle Autoritäten hauptsächlich über ihren praktischen Nutzen und ihre Fähigkeit, sozialen Zusammenhalt und gemeinschaftliches Handeln initiieren zu können. Kritiker*innen argumentieren, diese Perspektive berücksichtige nur unzureichend die historische Entwicklung traditioneller Herrschaft (Grischow 2008; Kleist 2011; Knierzinger 2011). Jeff Grischow (2008) verweist darauf, dass Verfechter*innen traditioneller Herrschaft sich tendenziell stärker auf den Zusammenhalt und die Harmonie innerhalb traditionell verwalteter Gemeinschaften konzentrierten, als auf Stratifizierung und Hierarchien. Dieser Trend in der Social Capital Literatur sei eng mit der Tendenz verflochten, durch einen a priori Fokus auf eine kohärente Gemeinschaft, bestehende Konflikte zu übersehen. Eine stärker historische Perspektive zeige, dass viele als traditionell bezeichnete Gemeinschaften stratifiziert, also durch tief verwurzelte Hierarchien sozialer und politischer Macht geprägt sind. Dies zu ignorieren, berge das Risiko, historisch geschaffene und gesellschaftlich verankerte Ungleichheit zu reproduzieren (Capps 2018). Johannes Knierzinger (2011) unterstreicht diesen Punkt. Er argumentiert, dass traditionelle Herrschaft vor allem ein Instrument der Elitenbildung sei und als solches durch vermeintliche kulturelle Werte abgesichert werde. Insbesondere beruhe es auf kulturellem und sozialem Kapital (vgl. Bourdieu 1986). Zu kulturellem Kapital zählen akademische Abschlüsse, Bildung und Wissen. Soziales Kapital kann über Adelstitel oder die Mitgliedschaft in sozialen Netzwerken erworben werden. Anhand einer Analyse zu Entwicklungsvermittler*innen in Ghana kommt er zu dem Schluss, dass 
die Fluidität und Verhandelbarkeit neotraditioneller Herrschaftssysteme es unterschiedlichen Akteuren erlaube, durch besonderes Engagement im Entwicklungsbereich den relativ neuen und daher auch als neotraditionell bezeichneten Titel des Entwicklungs-chiefs (chiefofdevelopment) zu erlangen. Dieser Titel ermögliche den Zugang zum traditionellen Herrschaftssystem, der immer auch mit gesellschaftlichem Aufstieg verbunden sei. Unter anderem erleichtere er den Kontakt zu Politiker*innen, internationalen Akteuren und erhöhe die Möglichkeiten ins Ausland zu gehen. Als problematisch erachtet Knierzinger, dass trotz des relativ einfachen Zugangs zu neotraditionellen Posten noch keine Entmystifizierung des chieftaincy-Systems stattgefunden habe. Ein Hinweis darauf sei die Beobachtung, dass die Macht von chiefs selten herausgefordert würde (ebd.: 41). Neotraditionelle Strukturen, die von außen nicht als solche erkannt würden, könnten zu einem Missbrauch traditioneller Systeme führen. Auch Nauja Kleist (2011) fordert eine stärkere Berücksichtigung neotraditioneller Entwicklungen in traditionellen Herrschaftssystemen. Anhand ihres Beitrags zu modernen chiefs in Ghana illustriert sie die Wandelbarkeit und Vielschichtigkeit traditioneller Herrschaft sowie die Entstehung neotraditioneller Handlungsmuster. Ebenso wie Knierzinger liegt ihr Fokus auf Immigrant"innen, die nach ihrer Rückkehr aus dem Ausland in Ämter traditioneller chiefs berufen werden. Kleist argumentiert, dass diese einen besonderen Status in der Gemeinschaft genössen, da sie mit ihrer internationalen Erfahrung und ihren Kontakten das Streben nach Entwicklung und Modernisierung verkörperten. Gleichzeitig sei es für sie jedoch auch besonders wichtig, ihre Legitimität in der Gemeinschaft zu festigen, indem sie unter anderem anhand von Kleidung, Bräuchen und Normen vermeintlich traditionelle Werte aufrechterhielten. Die Anerkennung und die damit verbundene Legitimität dieser neotraditionellen chiefs würden in einem Spannungsfeld von Tradition und Moderne verhandelt und inszeniert. Beide Sphären fänden gesellschaftlichen Anklang, weshalb neotraditionelle chiefs auch über die Fähigkeit verfügen müssten, sich in beiden positionieren zu können. In der Forschung zu traditionellen Herrschaftssystemen sei es daher von zentraler Bedeutung, das Handeln traditioneller Autoritäten in Bezug auf beide Sphären zu verstehen und es gleichzeitig als lokal und global zu fassen (ebd. : 647).

Wissenschaftliche Beiträge zu traditionellen Autoritäten als Intermediären lassen darauf schließen, dass internationale Geberorganisationen die vermittelnde Rolle traditioneller Autoritäten fördern, obwohl Wissenschaftler*innen diese kritisch hinterfragen. Kritiker"innen nehmen insbesondere das Verhältnis zwischen traditionellen Autoritäten und ihrer Gemeinschaft 
in den Blick. Sie hinterfragen, inwiefern traditionelle Autoritäten tatsächlich die Interessen ihrer Gemeinschaft repräsentieren und inwiefern Mitglieder der Gemeinschaft das Handeln traditioneller Autoritäten herausfordern (können). Im Zentrum der Kritik an traditioneller Herrschaft steht die mangelnde Rechenschaftspflicht traditioneller Autoritäten gegenüber ihrer Gemeinschaft, die oftmals mit intransparentem Handeln einhergeht. Hinsichtlich der entwicklungspolitischen Zusammenarbeit mit traditionellen Autoritäten als Intermediären zwischen ihrer Gemeinschaft und Dritten verweisen Kritiker*innen darüber hinaus auf die mangelnde Berücksichtigung der historischen Entwicklung traditioneller Herrschaft während der Kolonialzeit. Als Instrument der Elitenbildung berge traditionelle Herrschaft das Risiko zu einer Reproduktion und Perpetuierung historisch gewachsener und gesellschaftlich verankerter Macht- und Ungleichheitsverhältnisse zu führen und erweise sich in diesem Zuge zum Nachteil für schwächere Akteure. Mit meiner Forschung baue ich auf diese Erkenntnisse auf und berücksichtige insbesondere die verschiedenen Rollen traditioneller Autoritäten, die ihnen in unterschiedlichen Kontexten zugeschrieben wurden und seither parallel fortbestehen. In meiner Analyse des Handelns traditioneller Autoritäten in Konflikten um Zugang, die im Kontext von land grabbing auftreten, zeige ich inwiefern eine differenzierte Berücksichtigung dieser unterschiedlichen Rollen von Bedeutung für die Erklärung von Konflikten ist.

\subsection{Bezug zur bestehenden Forschung - Anknüpfungspunkte und Leerstellen}

Ziel des Kapitels war es, den aktuellen Stand der Forschung in Bezug auf traditionelle Autoritäten in Konflikten um Zugang zu Land, die im Zusammenhang mit land grabbing auftreten, aufzuarbeiten, zentrale Leerstellen zu identifizieren und Anknüpfungspunkte für meine eigene Forschung zu bestimmen. Dafür habe ich die bisherige Forschung zu (1) Konflikten im Zusammenhang mit land grabbing, (2) Land, sozialer Zugehörigkeit und Konflikten sowie (3) traditionellen Autoritäten als Intermediäre aufgearbeitet.

Aus den Arbeiten zu Konflikten im Kontext von land grabbing lässt sich ableiten, dass es im Kern dieser Konflikte um unterschiedliche Formen der Aushandlung von Zugang zu Land geht und dass Zugang zu Land wiederum eng mit bestehenden Machtverhältnissen zwischen den Konfliktakteuren verknüpft ist. Bislang konzentrieren sich Beiträge zur Analyse dieser Konflik- 
te auf drei Gruppen von Konfliktakteuren: Unternehmensakteuren, staatliche Akteure und die Landnutzer*innen. Die Rolle traditioneller Autoritäten in diesen Konflikten findet hingegen unzureichende Berücksichtigung. Dieser blinde Fleck in der Forschung zu Konflikten, die im Zusammenhang mit land grabbing auftreten, überrascht, da traditionellen Autoritäten in diesen Konflikten eine paradoxe Position einnehmen. Sie ermöglichen den Zugang $\mathrm{zu}$ Land für agrarindustrielle Vorhaben und sind gleichzeitig Vertreter*innen der Interessen ihrer Gemeinschaft. Zentrales Interesse vieler Mitglieder der Gemeinschaft ist jedoch der Zugang zu Land. Dieses Interesse steht im Widerspruch zur Implementierung agrarindustrieller Vorhaben, wie in Konflikten, die in diesem Kontext auftreten, deutlich wird. Über die Analyse des Handelns traditioneller Autoritäten im Rahmen großflächiger Landvergaben und damit einhergehender Konflikte möchte ich diese widersprüchliche Rolle traditioneller Autoritäten in den Blick nehmen und herausarbeiten, inwiefern sie für die Erklärung von Konflikten um Zugang in traditionellen Landrechtssystemen von Bedeutung ist. In meiner Analyse berücksichtige ich insbesondere die soziale Differenzierung innerhalb der Gemeinschaft, die sich über ungleiche Möglichkeiten des Zugangs zu Land ausdrückt. Mit meiner Forschung möchte ich zudem an die Debatte um die Ausgestaltung von Landrechten in traditionellen Landverwaltungssystemen anknüpfen.

Im Rahmen von Landreformen gibt es seit Beginn der $2000 e r$ Jahre eine Tendenz zur Formalisierung traditioneller Landrechte und der Integration von Gewohnheitsrecht in nationales Recht, um Zugang zu Land abzusichern (Chimhowu/Woodhouse 2006:347). Die Debatte darum, wie diese Integration aussehen soll, ist durch eine Dichotomie zwischen der Registrierung individueller Rechte und der Anerkennung kollektiver Rechte. Verfechter*innen der Formalisierung von Rechten beziehen sich insbesondere auf eine Studie von de Soto (2000), der die Mehrdeutigkeit und Verhandelbarkeit traditioneller Rechte als Ursachen für eine geringe Produktivität identifiziert. Die Registrierung von Landrechten war außerdem zentraler Bestandteil marktorientierter Agrarreformen, um den Zugang zu Land für Investoren zu erleichtern (Lahiff et al. 2007). Kritiker*innen dieser Bestrebungen argumentieren, die Registrierung von Landrechten wirke sich nachteilig auf diejenigen Akteure mit ohnehin schwachen Rechten an Land aus und blende sekundäre Rechte, wie die Weidenutzung und das Sammeln von Feuerholz oder wild vorkommender Nahrungsmittel aus (Toulmin 2009; Toulmin/Quan 2000). Folglich sprechen sie sich für die Anerkennung kollektiver Rechte aus. Dafür bedarf es jedoch der Berücksichtigung sozialer Differenzierung innerhalb lokaler Ge- 
meinschaften (Kirst/Prause 2019), die bislang in der Debatte um die Anerkennung kollektiver Rechte noch fehlt, jedoch relevant für die Absicherung des Zugangs zu Land derjenigen mit den schwächsten Rechten ist. Damit die Anerkennung traditioneller Rechte nicht zur Reproduktion sozialer Ungleichheit führt, bedarf es einer differenzierten Analyse von Machtverhältnissen, die in Konflikten um Zugang zu Land im Zusammenhang mit land grabbing besonders deutlich werden. Eine Analyse dieser Machtverhältnisse kann, neben einem besseren Verständnis von Konflikten um Zugang, dazu beitragen ein besseres Verständnis der Herausforderungen traditioneller Land- und Ressourcenverwaltung zu entwickeln und mögliche Potenziale, die sie mit sich bringen kann, zu erkennen.

Im folgenden Kapitel entwickle ich einen Analyserahmen, der es mir ermöglicht, den Zusammenhang zwischen dem Handeln traditioneller Autoritäten im Kontext von land grabbing, den damit einhergehenden Veränderung des Zugangs zu Land und den Konflikten, die in diesem Zusammenhang auftreten, zu untersuchen. 
